\title{
MODELING IRON ABUNDANCE ENHANCEMENTS IN THE SLOW SOLAR WIND
}

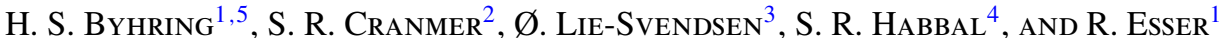 \\ ${ }^{1}$ Department of Physics and Technology, University of Troms $\emptyset$, NO-9037 Troms $\emptyset$, Norway; hanne-sigrun.byhring@uit.no \\ ${ }^{2}$ Harvard-Smithsonian Center for Astrophysics, 60 Garden Street, Cambridge, MA 02138, USA \\ ${ }^{3}$ Norwegian Defense Research Establishment, P.O. Box 25, NO-2027 Kjeller, Norway \\ ${ }^{4}$ Institute for Astronomy, University of Hawaii, 2680 Woodlawn Drive, Honolulu, HI 96822, USA \\ Received 2010 September 13; accepted 2011 March 9; published 2011 April 26
}

\begin{abstract}
We have studied the behavior of Fe ions in the slow solar wind, using a fluid model extending from the chromosphere to $1 \mathrm{AU}$. Emphasis is on elemental "pileup" in the corona, i.e., a region where the Fe density increases and has a local maximum. We study the behavior of individual $\mathrm{Fe}$ ions relative to each other in the pileup region, where $\mathrm{Fe}^{+10}$ and $\mathrm{Fe}^{+12}$ have been used as examples. We find that elemental pileups can occur for a variety of densities and temperatures in the corona. We also calculate the ion fractions and obtain estimates for the freezing-in distance of Fe in the slow solar wind. We find that the freezing-in distance for iron is high, between 3 and $11 R_{\odot}$, and that a high outflow velocity, of order $50-100 \mathrm{~km} \mathrm{~s}^{-1}$, in the region above the temperature maximum is needed to obtain ion fractions for $\mathrm{Fe}^{+10}$ and $\mathrm{Fe}^{+12}$ that are consistent with observations.
\end{abstract}

Key words: Sun: abundances - Sun: corona

Online-only material: color figures

\section{INTRODUCTION}

Over the years, there have been numerous theoretical investigations of the properties of minor ions in the high-speed solar wind from polar coronal holes (see, e.g., the reviews of Cranmer 2009; Marsch 2006; Hollweg \& Isenberg 2002, and references therein). The great interest in the properties of minor ions in the corona and the wind is motivated by the expectation that minor ion properties may provide important insight into the processes responsible for the heating of the corona. Previously, theoretical investigations of the effect of different heating rates on silicon and oxygen ions in polar coronal holes by Lie-Svendsen \& Esser (2005) have shown that when the heavy ions are no longer strongly coupled to the protons, abundance enhancements will occur in the corona if the ions are not sufficiently heated. The absence of such abundance enhancements in the observations places a lower limit on the heavy ion heating rate.

Recent solar eclipse observations by Habbal et al. (2007) of Fe XI $789.2 \mathrm{~nm}$ and Fe XIII $1074.7 \mathrm{~nm}$ spectral lines show evidence of local density enhancements in these ions relative to the electron density at heights of about $1.4-1.5 R_{\odot}$ from the solar center. The most pronounced density enhancements reported by Habbal et al. (2007) are found on the edges of streamers. The observations by Habbal et al. (2007) also revealed that density enhancements may sometimes be present in only one of the two observed iron ions.

The identification of the source regions of the slow solar wind remains one of the important unresolved issues in solar physics. Candidate source regions include open-field regions on streamer boundaries, defined by, e.g., Uzzo et al. (2006, 2007) as the streamer "legs," and open-field regions on the edges of coronal holes, adjacent to the streamer boundaries (e.g., Abbo et al. 2010; Antonucci et al. 2005, 2006). Abbo et al. (2010) showed that the plasma parameters, specifically the electron density and the $\mathrm{O}$ vi kinetic temperature, change from streamerlike (high density and low $\mathrm{O}$ vi kinetic temperature) close to the

\footnotetext{
5 This work was carried out during a visit at the Harvard-Smithsonian Center for Astrophysics, 60 Garden Street, Cambridge, MA 02138, USA.
}

bright edges of the streamer to coronal-hole-like (low density and high $\mathrm{O}$ vi temperature) in the open-field region surrounding the streamer. Given the uncertainty about the origin of the slow wind, we have chosen to explore two different models. One where the slow wind is assumed to originate in a region with high density and low proton temperature intended to resemble the streamer-edge plasma, and one where the slow wind is assumed to originate in a region with low density and a higher proton temperature representative of conditions on the edges of a coronal hole, adjacent to a streamer. Another reason for using two different background solutions, regardless of how important or unimportant we believe them to be as a source region for the solar wind, is the possibility it offers for studying the dependence of Fe abundance enhancements on the coronal plasma parameters.

The goals of this investigation are twofold. First, we wish to study elemental abundance variations of $\mathrm{Fe}$ in the corona for two different height profiles of the proton-electron plasma parameters. We will also study the "pileup" of $\mathrm{Fe}^{+10}$ and $\mathrm{Fe}^{+12}$ in detail, and compare synthesized Fe XI line/continuum ratios to those obtained by Habbal et al. (2007). Second, we wish to compare the ion fractions obtained with the two background solutions to observed $\mathrm{Fe}$ ion fractions in the slow solar wind and to estimate the freezing-in heights for the different charge states of Fe.

\section{THE NUMERICAL MODEL}

The model is based on the gyrotropic transport equations of Janse et al. (2005) and calculates the radial profile of the plasma density, the flow velocity, the parallel and perpendicular temperature, and the heat flux along the magnetic field for each particle species. For the iron ions we neglect the heat flux, since it is expected to be small. The model extends from the mid-chromosphere to $1 \mathrm{AU}$ and includes radiative energy losses as well as ionization and recombination processes. We do not assume ionization equilibrium. The only parameters that can be chosen freely are the heating rates for the particles and the shape of the magnetic field. The model builds on the numerical 

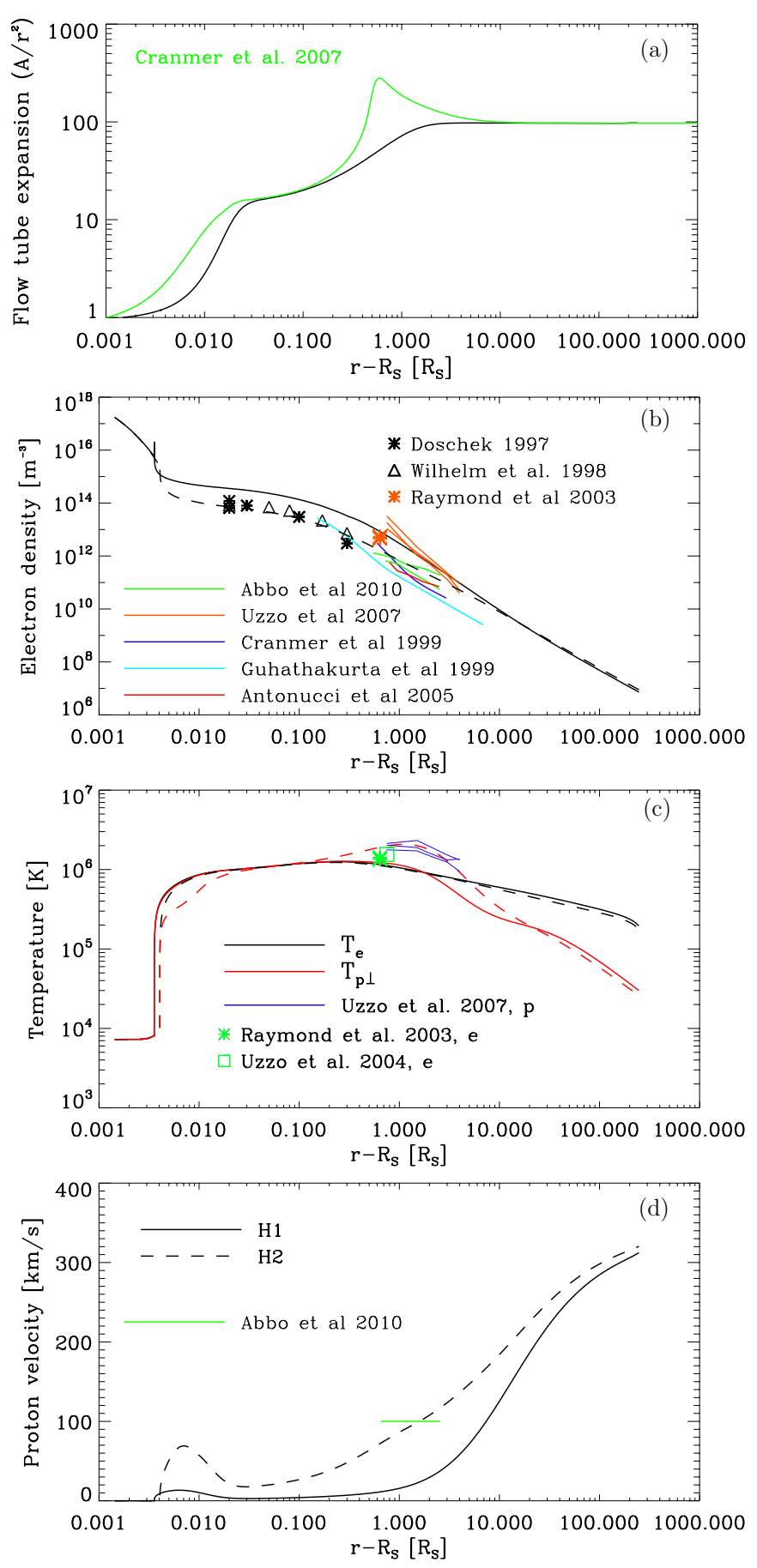

Figure 1. (a) Assumed flow tube expansion, $A / r^{2}$ both background solutions (black) and flow tube expansion assumed by Cranmer et al. (2007, green), (b) electron densities, (c) proton and electron temperatures, and (d) proton flow velocities for the hydrogen background solutions $\mathrm{H} 1$ (solid line) and $\mathrm{H} 2$ (dashed line).

(A color version of this figure is available in the online journal.)

model of Lie-Svendsen et al. (2001), and details of the numerical scheme, as well as information about the atomic data used in the hydrogen simulations, can be found in Lie-Svendsen \& Esser (2005, and references therein). The main difference between the model of Lie-Svendsen et al. (2001) and the model used here lies in an improved description of the Coulomb collisions, and hence of the heat conduction and thermal forces (Janse et al. 2005). The model equations can be found in Appendix A.
Table 1

Applied Energy Flux Densities at the Lower Boundary and the Resulting Proton Flux Densities at $1 \mathrm{AU}$ in $\mathrm{H} 1$ and H2

\begin{tabular}{lccc}
\hline \hline Solution & $\begin{array}{c}\text { Protons } \\
\left(\mathrm{W} \mathrm{m}^{-2}\right)\end{array}$ & $\begin{array}{c}\text { Electrons } \\
\left(\mathrm{W} \mathrm{m}^{-2}\right)\end{array}$ & $\begin{array}{c}\text { Proton Flux Density, } \\
1 \mathrm{AU}\left(10^{12} \mathrm{~m}^{-2} \mathrm{~s}^{-1}\right)\end{array}$ \\
\hline H1 & 2500 & 3300 & 3.0 \\
$\mathrm{H} 2$ & 3200 & 3300 & 3.8 \\
\hline
\end{tabular}

Note. The models are shown in Figure 1.

The equations are solved first for the hydrogen-protonelectron "background," in which the minor ions can be treated as test particles. The protons and electrons are heated by a prescribed energy flux which flows through the lower boundary of the model and is damped within $1 R_{\odot}$ above the solar surface. The prescribed energy flux has its maximum at the lower boundary and decays exponentially with distance from the solar surface (see also Lie-Svendsen et al. 2002).

For the minor ions we apply a given heating rate per particle defined as

$$
Q_{\mathrm{sm}}= \begin{cases}0 & r<r_{1}, \\ C n_{s} \frac{r-r_{1}}{r_{2}-r_{1}} \exp \left(-\frac{r-R_{\odot}}{H_{m}}\right) & r_{1}<r<r_{2}, \\ C n_{s} \exp \left(-\frac{r-R_{\odot}}{H_{m}}\right) & r>r_{2},\end{cases}
$$

where $R_{\odot}$ is the solar radius and $n_{s}$ is the density of species $s$. The minor ion heating begins at a heliocentric distance of $r=r_{1}=1.05 R_{\odot}$ and is ramped up linearly to its full value at $r=r_{2}=1.15 R_{\odot}$. This is done to avoid numerical problems which may arise when the heating is switched on suddenly. Above this height the heating is damped with a damping length of $H_{m}=1 R_{\odot}$. The heating rate coefficient, $C$, can be specified individually for all the different charge states. The minor ions are heated only in the perpendicular direction. We have included 16 iron charge states in our simulations, neutral $\mathrm{Fe}$ to $\mathrm{Fe}^{+15}$. The ion fraction for $\mathrm{Fe}^{+15}$ is everywhere below 0.001 in all our simulations, so the exclusion of the higher charge states is not expected to have any effect on the results.

The rates for the production and loss of the Fe charge states due to electron collisions and recombinations have been obtained from the High Altitude Observatory Spectral Diagnostic Package for emitted radiation (HAOS-DIAPER; Judge \& Meisner 1994).

\section{THE HYDROGEN BACKGROUND SOLUTIONS}

The superradial magnetic field expansion is the same for all hydrogen solutions and is shown in Figure 1(a) (black line). The flow tube area at the lower boundary is normalized to $1 \mathrm{~m}^{2}$. At large heights, this geometry resembles the streameredge field from the empirically derived solar-minimum field of Banaszkiewicz et al. (1998). A slightly modified version of that field was employed by Cranmer et al. (2007), and this geometry is also plotted in the top panel (green line). At low heights, this expansion rate is consistent with the idea of so-called coronal funnels (Dowdy et al. 1986).

In Table 1 we have summarized the input energy flux density in the two background solutions. In addition to the direct heating of the plasma particles, energy is also supplied to the plasma in the form of Alfvén wave momentum deposition. The Alfvén wave energy flux density at the lower boundary is $1000 \mathrm{~W} \mathrm{~m}^{-2}$ 
Table 2

Fe Models

\begin{tabular}{|c|c|c|c|c|c|c|c|c|}
\hline \multirow[b]{2}{*}{ H1 } & \multicolumn{2}{|c|}{$C_{A}\left(10^{-18} \mathrm{~W}\right)$} & \multicolumn{2}{|c|}{$C_{B}\left(10^{-18} \mathrm{~W}\right)$} & \multicolumn{2}{|c|}{$C_{C}\left(10^{-18} \mathrm{~W}\right)$} & \multicolumn{2}{|c|}{$C_{D}\left(10^{-18} \mathrm{~W}\right)$} \\
\hline & 1 & $1 \mathrm{~A}$ & 10 & 1B & 20 & $1 \mathrm{C}$ & 0 & $1 \mathrm{D}$ \\
\hline $\mathrm{H} 2$ & 3 & $2 \mathrm{~A}$ & 5 & $2 \mathrm{~B}$ & 10 & $2 \mathrm{C}$ & 0 & $2 \mathrm{D}$ \\
\hline
\end{tabular}

Note. In each column the heating rate coefficient, in units of $10^{-18} \mathrm{~W}$, is on the left and the name of the solution is on the right.

in both models. At $1 \mathrm{AU}$, the Alfvén wave energy flux has been reduced by about $95 \%$. Also listed in Table 1 are proton flux densities at $1 \mathrm{AU}$ derived from the model.

The first hydrogen solution (H1) has a high density and a low proton temperature. As we can see from panel (b) in Figure 1, the H1 density (black solid line) is higher than the densities observed by Raymond et al. (2003) and Abbo et al. (2010); however, it is well below the densities observed in this region in the study by Uzzo et al. (2007). In the region above $2 R_{\odot}$, the density of the $\mathrm{H} 1$ solution is close to that observed by Uzzo et al. (2007). The proton perpendicular temperature (solid red line in panel (c)) in $\mathrm{H} 1$ is lower than the observed kinetic temperatures in the corona. The kinetic temperature includes broadening by non-thermal processes; however, our calculations of the kinetic temperature, using the Alfvén wave amplitudes obtained in the model, suggest that this effect is not very strong in the region below $2-3 R_{\odot}$. This is consistent with the results of Esser et al. (1986). The electron temperature (black lines in panel (c) of Figure 1) is more or less identical in both background solutions, with a maximum of about $1.2 \mathrm{MK}$. The flow velocity in the corona is very low in H1 (panel (d) in Figure 1).

The second solution (H2) has a low density and a high proton temperature. In the low corona, below $2 R_{\odot}$, the density of $\mathrm{H} 2$ is clearly coronal-hole-like, as shown in Figure 1 where the $\mathrm{H} 2$ density is compared to observations from coronal holes by Doschek (1997), Wilhelm et al. (1998), Cranmer et al. (1999), and Guhathakurta et al. (1999). In the region 1.6-3.5 $R_{\odot}$, the density of $\mathrm{H} 2$ is close to that of Abbo et al. (2010). The proton perpendicular temperature is higher in the $\mathrm{H} 2$ solution than in the $\mathrm{H} 1$ solution. The maximum proton perpendicular temperature in $\mathrm{H} 2$ is $2 \mathrm{MK}$, which is in good accordance with the results of Uzzo et al. (2007), but somewhat higher than the temperatures observed by Abbo et al. (2010) and Antonucci et al. (2005), who obtained proton kinetic temperatures just below $2 \mathrm{MK}$. The flow velocity in the corona is higher in $\mathrm{H} 2$ than in $\mathrm{H} 1$. Abbo et al. (2010) found flow velocities of about $100 \mathrm{~km} \mathrm{~s}^{-1}$ in the region between 1.6 and $3.5 R_{\odot}$ and this is in good accordance with the flow velocity obtained in $\mathrm{H} 2$. The peak in the flow velocity below $1.2 R_{\odot}$ is a consequence of the funnel shape of the flow tube.

\section{IRON SIMULATION RESULTS}

For both hydrogen solutions, the model equations (see Appendix A) are solved for all $16 \mathrm{Fe}$ charge states simultaneously, treating the Fe ions as test particles in a fixed background. The fractional abundance of $\mathrm{Fe}$, relative to hydrogen, at the lower boundary has been set to $3 \times 10^{-5}$. For both hydrogen backgrounds we have chosen four different values of the heating rate coefficient, $C$, which specifies the maximum heating rate for the Fe ions (see Equation (1)). This means that we have produced eight different $\mathrm{Fe}$ solutions, which are summarized in Table 2.

In the following sections, we will discuss two types of coupling between the $\mathrm{Fe}$ ions and the background proton-electron plasma. The first type is the collisional coupling between Fe ions and protons. As the proton density falls off in the corona, the collisional coupling between protons and Fe decreases allowing the Fe temperature and flow velocity to deviate from the proton temperature and flow velocity. This occurs somewhere below $2 R_{\odot}$ and is referred to in the text as "decoupling."

The second type of coupling is related to ionization and recombination of Fe ions. As the Fe velocity increases in the extended corona, the timescale for expansion decreases and eventually drops below the timescales for ionization and recombination (in which collisions with electrons is an important factor). The Fe ions finally reach a state where the flux of each individual charge state is constant with respect to $r$ (Hundhausen et al. 1968). This occurs somewhere above $2 R_{\odot}$ and is referred to as "freezing-in" (see Section 4.4 for the results on the freezing-in distance of $\mathrm{Fe}$ ).

\subsection{Average Properties}

The Fe flux into the corona is determined by the $\mathrm{Fe}$ abundance and flow velocity at the base of the transition region. As discussed by Lie-Svendsen \& Esser (2005), no coronal process can regulate the flux of minor ions into the corona, because the thermal and frictional forces on the minor ions are very large in the transition region. If the $\mathrm{Fe}$ ions do not have enough energy when they decouple from the protons in the corona, they will not be able to escape the solar gravitational field and they will be trapped in the region where they decouple. And since the supply of Fe from below is not affected by what goes on in the corona, the $\mathrm{Fe}$ abundance will increase in the decoupling region. As the Fe density increases, a pressure gradient force is established in the direction away from the Sun, eventually pushing the Fe ions out of the decoupling region. A steady state is reached when the force balance in the corona has been adjusted such that the $\mathrm{Fe}$ flux out of the corona matches the Fe flux into the corona.

In Figure 2 we show the average properties of $\mathrm{Fe}$ for heating rates $C_{B}$ and $C_{D}$ in $\mathrm{H} 1$ and $\mathrm{H} 2$. The total Fe density $\left(n_{\mathrm{Fe}}\right)$ is defined as

$$
n_{\mathrm{Fe}}=\sum_{s} n_{s}
$$

and the average Fe temperature $\left(T_{\mathrm{Fe}}\right)$ and velocity $\left(u_{\mathrm{Fe}}\right)$ are defined as

$$
\begin{aligned}
& T_{\mathrm{Fe}}=\frac{1}{n_{\mathrm{Fe}}} \sum_{s} n_{s} \cdot T_{s}, \\
& u_{\mathrm{Fe}}=\frac{1}{n_{\mathrm{Fe}}} \sum_{s} n_{s} \cdot u_{s},
\end{aligned}
$$

where the sum is over all charge states of $\mathrm{Fe}$ and $T_{s}=$ $\left(T_{s, \|}+2 T_{s, \perp}\right) / 3$ is the average temperature of species $s$. In the following, we will use the subscript $s$ for quantities associated with an individual $\mathrm{Fe}$ species and the subscript $\mathrm{Fe}$ for quantities that have been calculated by averaging over all Fe species.

Although the pressure gradient force is what ultimately ensures a force balance where the Fe flux out of the corona is 

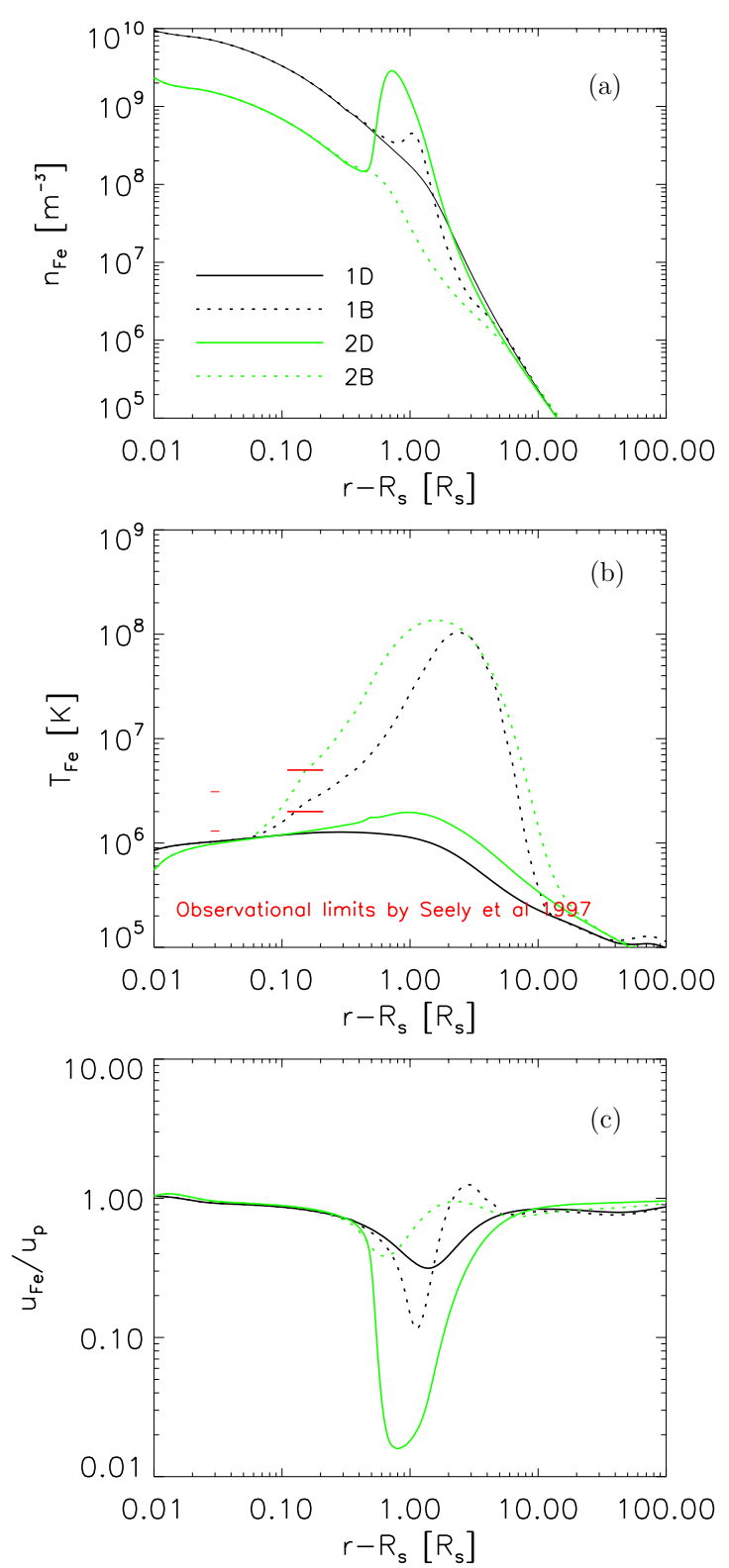

Figure 2. (a) Total iron density, (b) average iron temperature, and (c) $u_{\mathrm{Fe}} / u_{p}$ for iron solutions 1-2B and 1-2D (see Table 2). In solutions 1-2D the iron temperature is identical to the average proton temperature. The observational limits shown in (b) are from Seely et al. (1997).

(A color version of this figure is available in the online journal.)

the same as the Fe flux into the corona, it is not necessarily the dominant force on the Fe ions in this region. For our background solutions, in the case where no heating is applied to the Fe ions (corresponding to solutions $1 \mathrm{D}$ and $2 \mathrm{D}$ in Figure 2), the $\mathrm{Fe}$ velocity in the decoupling region is determined mainly through the force balance between friction and gravity

$$
\frac{G M_{\odot}}{r^{2}}=g=v_{\mathrm{FeP}}\left(u_{p}-u_{\mathrm{Fe}}\right) \Rightarrow u_{\mathrm{Fe}}=u_{p}-\frac{g}{v_{\mathrm{FeP}}},
$$

where $G$ is the gravitational constant, $M_{\odot}$ is the solar mass, and $\nu_{\mathrm{FeP}}=n_{p} \cdot \gamma_{\mathrm{FeP}}$ is the average collision frequency between Fe and protons. The conservation of proton and $\mathrm{Fe}$ flux requires that

$$
\frac{n_{\mathrm{Fe}}}{n_{p}}=K \frac{u_{p}}{u_{\mathrm{Fe}}}=\frac{K}{1-\frac{g}{u_{p} n_{p} \gamma_{\mathrm{FeP}}}},
$$

where $K$ is a proportionality constant (i.e., the Fe abundance at the lower boundary). The pressure gradient force will only be responsible for a small correction to the above expression. The magnitude of the abundance enhancement, or "pileup" (i.e., $n_{\mathrm{Fe}} / n_{p}$, from the above equation), is thus determined mainly by the proton flux density, $n_{p} u_{p}$, and $\gamma_{\mathrm{FeP}}=v_{\mathrm{FeP}} / n_{p}$. The average collision frequency, $v_{\mathrm{FeP}}$, is

$$
v_{\mathrm{FeP}}=\frac{1}{n_{\mathrm{Fe}}} \sum_{s} n_{s} \cdot v_{s p},
$$

where $v_{s p}$, the Coulomb collision frequency between an individual Fe species (s) and protons, is

$$
v_{s p}=\frac{1}{3} \frac{n_{p} m_{p}}{m_{s}+m_{p}}\left(\frac{2 \pi k T_{s p}}{\mu_{s p}}\right)^{-3 / 2} \frac{Z^{2} e^{4}}{\epsilon_{0}^{2} \mu_{s p}^{2}} \ln \lambda,
$$

where $T_{s p}=\left(T_{s} m_{p}+T_{p} m_{s}\right) /\left(m_{s}+m_{p}\right)$ is the reduced temperature, $\mu_{s p}=m_{s} m_{p} /\left(m_{s}+m_{p}\right)$ is the reduced mass, $\epsilon_{0}$ is the permittivity of vacuum, $\ln \lambda$ is the Coulomb logarithm, $k$ is the Boltzmann constant, $e$ is the elementary charge, and $Z e$ is the charge of the $\mathrm{Fe}$ ion. From the above expressions we can see that $\gamma_{\mathrm{FeP}}=v_{\mathrm{FeP}} / n_{p}$ depends mainly on the average proton temperature (i.e., $\left.T_{p}=\left(T_{p, \|}+2 T_{p, \perp}\right) / 3\right)$, but also weakly on the Fe temperature $\left(T_{s p} \approx T_{p}+\left(m_{p} / m_{s}\right) T_{s}\right.$, since $m_{s}$ is much larger than $m_{p}$ ).

In $\mathrm{H} 1$, the largest pileup occurs in solution 1B (panel (a), Figure 2, see also Figure 4). As a result of a very low flow velocity, the $\mathrm{H} 1$ solution has a low proton flux density, which should favor a large pileup (cf. Equation (6)). However, the H1 background is so cool, that for low heating rates (i.e., 1D and 1A, Table 2) the Fe ions are collisionally coupled to the protons all the way through the corona. The Fe ions are only able to decouple from the protons when the Fe temperature reaches a certain level. The Fe temperature required for decoupling depends on the background density and temperature. Thus, the 1D solution shows that in a low temperature background, such as $\mathrm{H} 1$, it is possible for Fe to escape from the corona without being heated and without piling up.

In $\mathrm{H} 2$, on the other hand, the largest pileup occurs when the $\mathrm{Fe}$ ions are not heated, i.e., in 2D (green solid line in Figure 2, see also Figure 4). This is because the coupling to the protons is weaker in $\mathrm{H} 2$ than in $\mathrm{H} 1$, as a result of the higher proton temperature in $\mathrm{H} 2$. In this background the exact position of the Fe pileup is determined by the proton temperature and flux density. For a given flux density, a small reduction in temperature will cause the pileup to move to a higher altitude (and a small temperature increase will cause the pileup to occur further down).

The $1 \mathrm{D}$ and $2 \mathrm{D}$ solutions show that in the slow solar wind $\mathrm{Fe}$ can escape from the corona without being heated, i.e., with an $\mathrm{Fe}$ temperature which is only of the order of the proton temperature (Figure 2(b)). This is in contrast to the result obtained for the high-speed solar wind by Lie-Svendsen \& Esser (2005).

From panel (c) in Figure 2 we see that in the region between 1.5 and $6 R_{\odot}$, the average Fe flow speed deviates significantly from the proton flow speed. However, above $6 R_{\odot}$ the Fe ions are re-coupled to the protons and are able to escape the solar gravitational field (they reach $u_{\text {esc }}$ at $\approx 10-15 R_{\odot}$ ) because of friction with the protons.

\subsection{Pileup of $\mathrm{Fe}^{+10}$ and $\mathrm{Fe}^{+12}$}

So far, we have been concerned only with elemental pileup, where the abundance of an element increases in some region of 

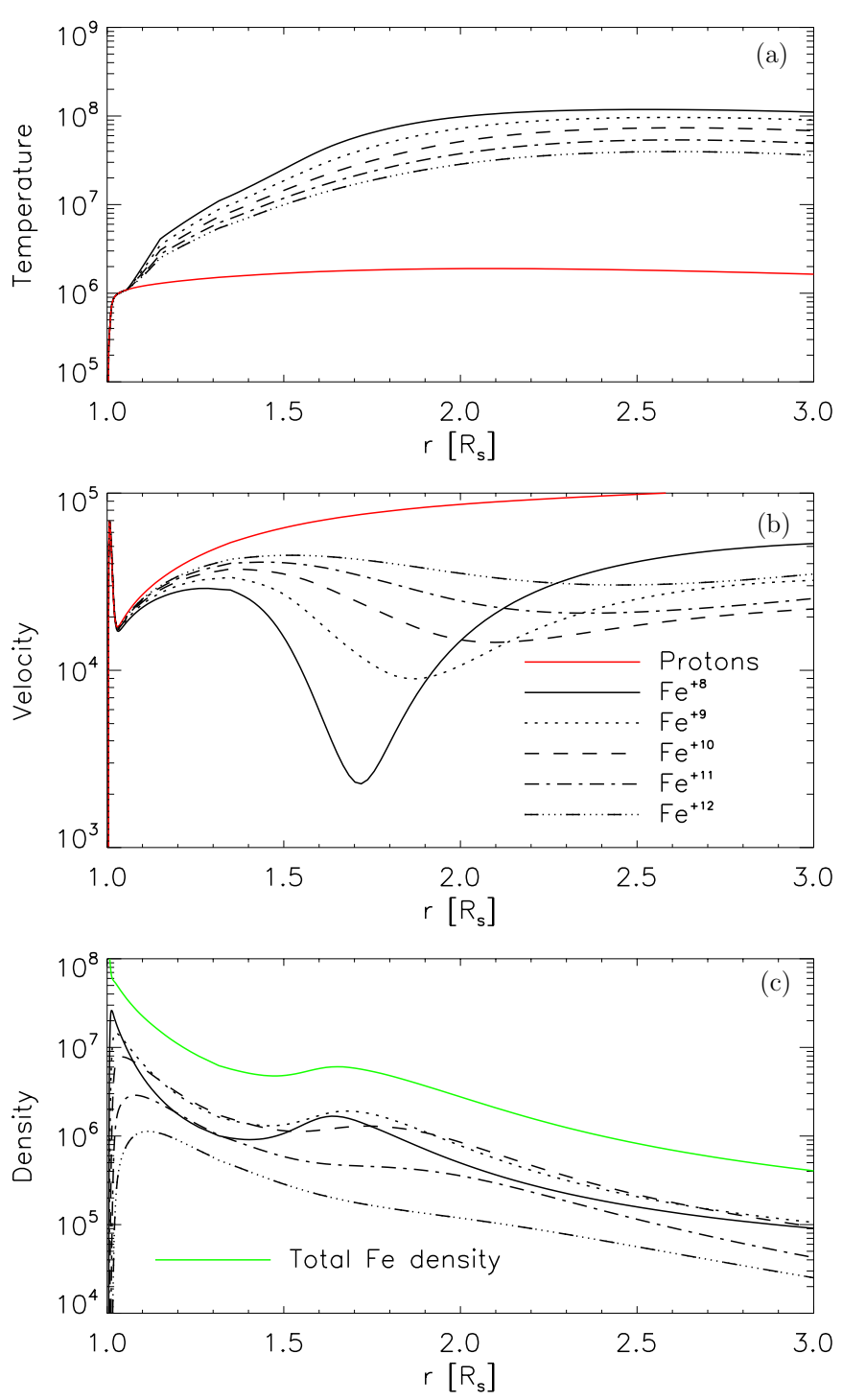

Figure 3. (a) Temperature, (b) velocity, and (c) density of $\mathrm{Fe}^{+8}-\mathrm{Fe}^{+12}$ in iron solution $2 \mathrm{~A}$ (see Table 2).

(A color version of this figure is available in the online journal.)

the corona. We also wish to study the densities of the individual Fe charge states. From the expression for the Coulomb collision frequency between an individual Fe species and protons (Equation (8)), it is evident that the coupling to the background for an individual $\mathrm{Fe}$ species depends on the ion charge, as well as the (average) temperature of that particular species. This means that the degree of pileup may vary from charge state to charge state.

To see this, we have plotted the average temperature (i.e., $T_{s}=$ $\left.\left(T_{s, \|}+2 T_{s, \perp}\right) / 3\right)$, the velocity and the density of $\mathrm{Fe}^{+8}-\mathrm{Fe}^{+12}$ for model $2 \mathrm{~A}$ in Figure 3. Also shown are the average proton temperature and flow velocity (red lines) and the total Fe density (green line). We find that the highest temperature (of those shown in the figure) is reached by $\mathrm{Fe}^{+8}$, followed by $\mathrm{Fe}^{+9}$, then $\mathrm{Fe}^{+10}$, and so on. $\mathrm{Fe}^{+8}$ also has the largest drop in flow velocity, relative to the protons. The largest pileup is seen in $\mathrm{Fe}^{+8}$, followed by $\mathrm{Fe}^{+9}$, and then $\mathrm{Fe}^{+10}$. In $\mathrm{Fe}^{+12}$, no increase in the density can be seen, even though the $\mathrm{Fe}^{+12}$ flow velocity does drop below that of the protons. Since the heating rate is the same for all charge states, these are all direct results of the charge dependency of the Coulomb collision frequency.
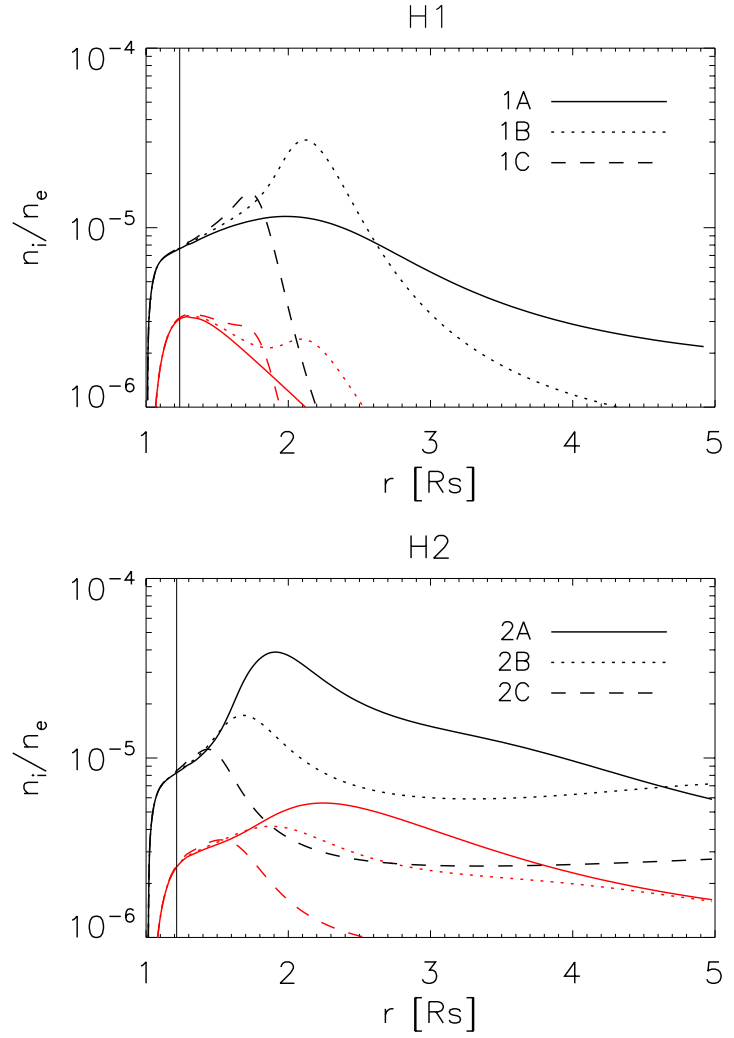

Figure 4. $n_{i} / n_{e}$ for $\mathrm{Fe}^{+10}$ (black) and $\mathrm{Fe}^{+12}$ (red) for different values of the heating rate coefficient, $C$ (see Table 2), in both hydrogen backgrounds.

(A color version of this figure is available in the online journal.)

In Figure 4, we have plotted $n_{i} / n_{e}$ for $\mathrm{Fe}^{+10}$ and $\mathrm{Fe}^{+12}$ for solutions $1 \mathrm{~A}-\mathrm{C}$ and $2 \mathrm{~A}-\mathrm{C}$. In ionization equilibrium, the ion fractions of $\mathrm{Fe}^{+10}$ and $\mathrm{Fe}^{+12}$ reach their highest values at a temperature equal to, or higher than, the maximum electron temperature obtained in our solutions. This means that any increase in $n_{i} / n_{e}$ that occurs above the electron temperature maximum (at $1.2 R_{\odot}$, indicated by vertical lines in the figure) is a pileup. We find that the solutions $1 \mathrm{~B}$ and $2 \mathrm{~A}$ have fairly large pileups in both $\mathrm{Fe}^{+10}$ and $\mathrm{Fe}^{+12}$. The $1 \mathrm{C}$ and $2 \mathrm{~B}$ solutions also have clearly visible pileups in the $\mathrm{Fe}^{+10}$ density, but not in the $\mathrm{Fe}^{+12}$ density.

In the observations of Habbal et al. (2007), a density enhancement is sometimes seen only in the Fe XI $789.2 \mathrm{~nm}$ line, and not in the Fe XIII $1074.7 \mathrm{~nm}$ line. This could be an effect of preferential heating of $\mathrm{Fe}^{+12}$ relative to $\mathrm{Fe}^{+10}$. We test this idea by redoing the simulation for $\mathrm{H} 2$ in which a large pileup was obtained in $\mathrm{Fe}^{+10}$ and $\mathrm{Fe}^{+12}$ (i.e., 2A), but now we heat the $\mathrm{Fe}^{+12}$ more than the $\mathrm{Fe}^{+10}$. Since the ion fractions of all charge states above $\mathrm{Fe}^{+9}$ are decreasing in the pileup region, which means that the source term in the equations for $\mathrm{Fe}^{+12}$ that results from recombination of $\mathrm{Fe}^{+13}$ is important in this region, we have chosen to apply extra heating not only to $\mathrm{Fe}^{+12}$, but also to $\mathrm{Fe}^{+13}$. The result can be seen in Figure 5. We find that the preferential heating of $\mathrm{Fe}^{+12}$ and $\mathrm{Fe}^{+13}$ causes the pileup in $\mathrm{Fe}^{+12}$ to disappear, while the pileup in $\mathrm{Fe}^{+10}$ is retained.

\subsection{Comparison with Observations of Fe Spectral Lines}

Figure 6(a) shows synthesized Fe XI 7892 line/continuum ratios for the $\mathrm{H} 2$ background. The ratios have been normalized to 1 (a description of the Fe XI $789.2 \mathrm{~nm}$ line calculation can be found in Appendix B). Thus, we can only compare the shapes 


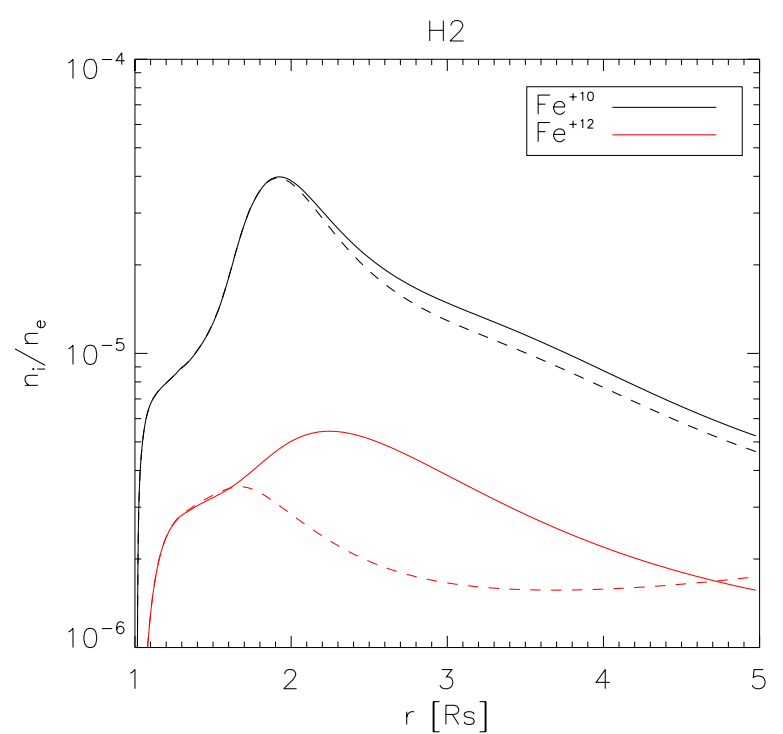

Figure 5. $n_{i} / n_{e}$ for $2 \mathrm{~A}$ with (dashed lines) and without (solid lines) increased heating of $\mathrm{Fe}^{+12}$ and $\mathrm{Fe}^{+13}$. An increased heating rate of $C=10^{-17} \mathrm{~W}$ was used for $\mathrm{Fe}^{+12}$ and $\mathrm{Fe}^{+13}$

(A color version of this figure is available in the online journal.)
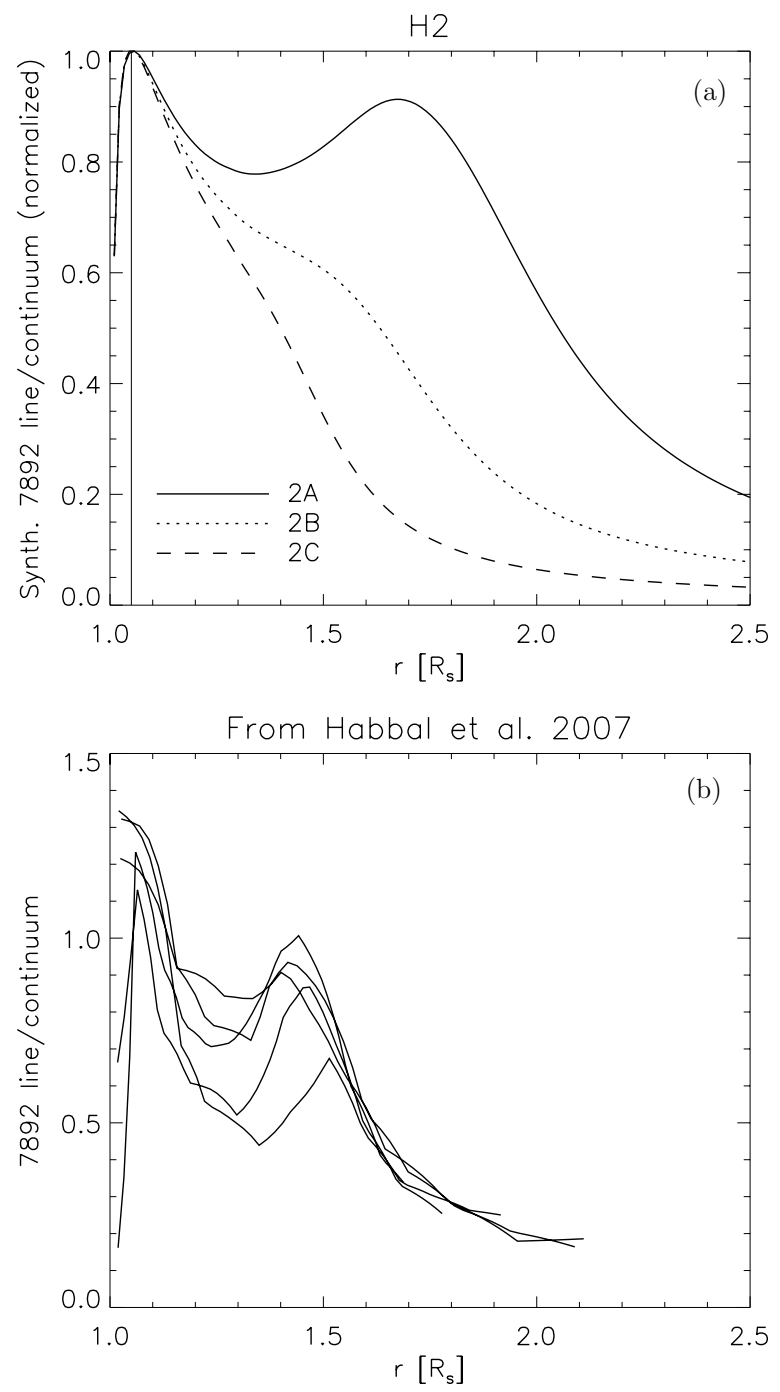

Figure 6. (a) Synthesized Fe XI $789.2 \mathrm{~nm}$ line/continuum ratio for three different values of the $\mathrm{Fe}$ heating rate, $C$, in the $\mathrm{H} 2$ background and (b) observed $\mathrm{Fe}$ XI line/continuum ratios from Habbal et al. (2007, their Figure 9, left panel). In panel (a), a vertical black line has been drawn at the height $1.05 R_{\odot}$.
Table 3

Ion Fractions, at the Height of the Temperature Maximum and at $1 \mathrm{AU}$, and Freezing-in Heights for $\mathrm{Fe}^{+10}$ and $\mathrm{Fe}^{+12}$ in Six Fe Solutions (see also Table 2)

\begin{tabular}{|c|c|c|c|c|c|c|}
\hline \multirow[t]{3}{*}{ Fe solution } & \multicolumn{3}{|c|}{$\mathrm{Fe}^{+10}$} & \multicolumn{3}{|c|}{$\mathrm{Fe}^{+12}$} \\
\hline & \multicolumn{2}{|c|}{ Ion Fraction } & \multirow[t]{2}{*}{$r_{\text {freeze }}\left(R_{\odot}\right)$} & \multicolumn{2}{|c|}{ Ion Fraction } & \multirow[t]{2}{*}{$r_{\text {freeze }}\left(R_{\odot}\right)$} \\
\hline & $T_{e}^{\max }$ & $1 \mathrm{AU}$ & & $T_{e}^{\max }$ & $1 \mathrm{AU}$ & \\
\hline $1 \mathrm{~A}$ & 0.28 & 0.06 & 11 & 0.13 & 0.003 & 9.5 \\
\hline 1B & 0.28 & 0.02 & 10.3 & 0.13 & 0.003 & 9.5 \\
\hline $1 \mathrm{C}$ & 0.28 & 0.04 & 11.3 & 0.13 & 0.0002 & 7.7 \\
\hline $2 \mathrm{~A}^{*}$ & 0.28 & 0.09 & 7.4 & 0.09 & 0.04 & 8.2 \\
\hline $2 \mathrm{~B}^{*}$ & 0.28 & 0.16 & 7.2 & 0.09 & 0.04 & 6.7 \\
\hline $2 \mathrm{C}$ & 0.29 & 0.21 & 3.7 & 0.09 & 0.05 & 7.2 \\
\hline
\end{tabular}

Note. In solutions $2 \mathrm{~A}$ and $2 \mathrm{~B}$ (marked by an asterisk), there is a secondary pileup in the extended corona $\left(4-10 R_{\odot}\right)$.

of the calculated and measured line/continuum ratios, not their absolute values. Below $1.05 R_{\odot}$ (black vertical line in Figure 6), the ratio is increasing due to production of $\mathrm{Fe}^{+10}$ in the low corona. Between $1.05 R_{\odot}$ and $1.3 R_{\odot}$ the ratio drops off. We get a clear maximum in the line/continuum ratio at about $1.7 R_{\odot}$ for the lowest heating rate. For the two higher heating rates, the line/continuum ratio has a change in the slope starting at around $1.3 R_{\odot}$, but no peak. If we compare the line/continuum ratios with the corresponding ion/electron density ratios in Figure 4, we find that a rather large pileup is needed to produce a peak in the line/continuum ratio. This is a result, at least in part, of the contributions along the line of sight (LOS) from regions further away from the Sun. This can be seen by choosing a shorter LOS in the calculation of the spectral line (see Appendix B for details of the spectral line calculation). The synthesized line/continuum ratios look qualitatively similar to the line/continuum ratios shown in Figure 9 of Habbal et al. (2007), which we have plotted in Figure 6(b).

\subsection{Ion Fractions and Freezing-in}

Next, we wish to examine the ion fractions and freezing-in heights for $\mathrm{Fe}^{+10}$ and $\mathrm{Fe}^{+12}$. The electron temperature profile is more or less identical for both hydrogen background solutions. This means that any difference in the "frozen-in" ion fractions at $1 \mathrm{AU}$ between $\mathrm{H} 1$ and $\mathrm{H} 2$ is the result of the difference in flow speed, since the flow speed determines the degree of departure from ionization equilibrium and the height where freezing-in occurs. Generally, a high ion velocity will lead to large departures from ionization equilibrium, and freezing-in close to the Sun.

In the low-velocity background $\mathrm{H} 1$, the ion fractions are nearly identical to the ionization equilibrium values below $1.5 R_{\odot}$. Above this height they deviate slightly from the ionization equilibrium values. For the $\mathrm{H} 2$ background, the ion fractions are $5 \%-10 \%$ below the ionization equilibrium values at $1.2 R_{\odot}$, and above this height the departure from ionization equilibrium is large.

In Table 3 we have listed the freezing-in heights for $\mathrm{Fe}^{+10}$ and $\mathrm{Fe}^{+12}$, defined as the height above which the relative ion flux (i.e., $n_{i} u_{i} / n_{\mathrm{Fe}} u_{\mathrm{Fe}}$ ) is always within $10 \%$ of its value at $1 \mathrm{AU}$, for all Fe solutions shown in Figure 4. Also listed in Table 3 are the ion fractions for $\mathrm{Fe}^{+10}$ and $\mathrm{Fe}^{+12}$ at the height of the electron temperature maximum and at $1 \mathrm{AU}$. As we can see from the table, the freezing-in height is very large in most of the $\mathrm{Fe}$ solutions. For the high-speed solar wind, freezing-in distances of between 1.3 and $4 R_{\odot}$ have been reported (Chen et al. 2003; 
Ko et al. 1997). The lowest freezing-in height for $\mathrm{Fe}^{+10}$ and $\mathrm{Fe}^{+12}$ obtained in these simulations is $3.7 R_{\odot}$ (in solution $2 \mathrm{C}$ ).

The large freezing-in height means that the final frozen-in charge states are quite sensitive to the outflow velocity in the region above the temperature maximum. As we can see from the table, the ion fraction of $\mathrm{Fe}^{+12}$ at the height of the temperature maximum is higher in $\mathrm{H} 1$ compared to $\mathrm{H} 2$. However, at $1 \mathrm{AU}$ the ion fraction of $\mathrm{Fe}^{+12}$ in $\mathrm{H} 1$ is extremely low. Clearly, the low outflow velocity in this solution ensures that as the electron temperature drops, above $1.2 R_{\odot}$, the ion fractions of the higher charge states decrease considerably before they reach the freezing-in distance. In $\mathrm{H} 2$ the ion fraction of $\mathrm{Fe}^{+12}$ is much higher at $1 \mathrm{AU}$, because the high flow velocity keeps the Fe ions well out of ionization equilibrium. Two of the solutions should be mentioned in particular: $2 \mathrm{~A}$ and $2 \mathrm{~B}$. Solution $2 \mathrm{~A}$ displays a large secondary abundance enhancement in the extended corona $\left(4-10 R_{\odot}\right)$. In solution $2 \mathrm{~B}$ there is also a secondary pileup region, but it is less pronounced and mainly visible in the densities of the lower charge states. Nevertheless, in $2 \mathrm{~B}$ as well as in $2 \mathrm{~A}$ the secondary pileup has a large effect on the ion fractions at 1 AU. This is because the pileup is associated with a drop in the flow velocity. When the flow velocity falls off, the Fe ions come closer to being in ionization equilibrium, and since this occurs in a region of decreasing electron temperature, it leads to an increase in the ion fractions of the lower charge states at the expense of $\mathrm{Fe}^{+10}$ and $\mathrm{Fe}^{+12}$. However, keeping that in mind, it appears that it is the low density background, H2, which can produce ion fractions that are closest to the results of von Steiger et al. (2000), who found that the ion fractions for $\mathrm{Fe}^{+10}$ and $\mathrm{Fe}^{+12}$ in the slow solar wind are $0.2-0.25$ and $0.08-0.1$, respectively. The agreement between solution $2 \mathrm{C}$, in particular, and the results of von Steiger et al. (2000) is quite good, especially taken into account the uncertainties in the atomic data used in the model.

\section{DISCUSSION}

We have found that Fe pileups in the corona can occur for a range of proton densities and temperatures. Even in a solar wind with a strong coupling between protons and Fe, such as the $\mathrm{H} 1$ solution, pileup can occur if the Fe temperature exceeds some minimum value, (typically around $10 \mathrm{MK}$ in the $\mathrm{H} 1$ background) which depends on the density and temperature of the background, as well as the applied heating of the Fe ions. We find that the amount of heating that is consistent with a moderate pileup differs considerably between the two background models. This reflects the differences in density and temperature between the hydrogen backgrounds. Based on the results described here we cannot place any strong constraint on the required level of Fe heating in the corona.

As seen in Fe solutions 1-2D, the good coupling between protons and $\mathrm{Fe}$ in the slow solar wind ensures that it is not necessary to heat the Fe ions to temperatures of order $100 \mathrm{MK}$ for them to escape from the solar gravitational field. Even if Fe decouples from the protons and piles up in the corona, the Fe ions will re-couple to the protons a little further out (in the extended corona) and be dragged out by friction with protons. In a low density background such as $\mathrm{H} 2$, a large pileup is needed to push the Fe out of the pileup region and into the extended corona.

Our choice of magnetic field expansion is consistent with the idea of so-called coronal funnels (Dowdy et al. 1986) and resembles the streamer-edge field from the empirically derived solar-minimum field of Banaszkiewicz et al. (1998) at large heights. However, it does not have the characteristic expansion-contraction, seen just below $2 R_{\odot}$ in the field employed by Cranmer et al. (2007) in Figure 1 . The main effect of such an expansion-contraction of the magnetic field is a corresponding dip in the proton flux density. As discussed in Section 4.1, the magnitude of the pileup depends on the proton flux density, where a low flux density leads to larger pileups. Thus, we expect that choosing a magnetic field expansion such as that of Cranmer et al. (2007) might result in larger pileups than what was found in this study. It might also be possible to regulate the location of the pileup by adjusting the location of the expansion-contraction in the magnetic field. Nevertheless, the results obtained in this study are not expected to depend significantly on the choice of magnetic field expansion.

Habbal et al. (2007) found that enhancements in the $\mathrm{Fe}^{+12}$ density were not always present where $\mathrm{Fe}^{+10}$ density enhancements were observed. They suggested that this might be the result of a low electron temperature, favoring the production of the lower charge state. Since all of our hydrogen background solutions have approximately identical electron temperature profiles, we cannot test this idea; however, we do note that, although the electron temperatures in our simulations are close to the lower limit of observed values, $\mathrm{Fe}^{+12}$ is produced in both background solutions. In general, the amount of $\mathrm{Fe}^{+12}$ in the corona is determined not only by the magnitude of the electron temperature maximum, but also by the flow velocity in the region below the electron temperature maximum (where high velocities lead to less $\mathrm{Fe}^{+12}$ ), the flow velocity in the region above the electron temperature maximum (where high velocities lead to more $\mathrm{Fe}^{+12}$ ) and the rate of decrease of $T_{e}$ above the electron temperature maximum (Ko et al. 1997; Esser et al. 1998). In addition, the height where pileup occurs, which is determined mainly by the average proton temperature, could be important in determining whether or not enough $\mathrm{Fe}^{+12}$ is present to produce a significant pileup. We have also found that it is possible, by applying more heating to the higher charge states (i.e., $\mathrm{Fe}^{+12}$ and $\mathrm{Fe}^{+13}$ in Figure 5), to avoid a pileup in $\mathrm{Fe}^{+12}$, while still retaining a pileup in $\mathrm{Fe}^{+10}$. In summary, the presence of a pileup in $\mathrm{Fe}^{+12}$ seems to be a rather complicated function of the background plasma parameters and may also be affected by the amount of energy supplied to the higher charge states.

The synthesized line/continuum ratio of model $2 \mathrm{~A}$ and $2 \mathrm{~B}$ in Figure 6 quite successfully reproduces the observations by Habbal et al. (2007). This confirms that the observed "bumps" in the line/continuum ratio must be a signature of a local increase in the total density of $\mathrm{Fe}$, since ionization and recombination processes are not able to produce such increases in the density of a single charge state (or two). We find that a rather large pileup is needed to produce a local maximum in the line/continuum ratio.

Habbal et al. (2010) argued that when the Fe XI $789.2 \mathrm{~nm}$ line changes from the collisionally excited regime, where it is proportional to $n_{i} \cdot n_{e}$, to the radiatively excited regime, where it is proportional to $n_{i}$, the line/continuum ratio should change from rapidly decreasing (i.e., proportional to $n_{i}$ ) to a constant value (i.e., proportional to $n_{i} / n_{e}$ ), given that the radial falloff of ion and electron densities is the same. They named the distance above which the Fe XI $\lambda 789.2 \mathrm{~nm}$ line is dominated by radiative processes $R_{t}$ and estimated it from their data by finding the distance where the derivative of the line/continuum ratio becomes (nearly) zero. $R_{t}$, as determined by Habbal et al. (2010), varied between 1.1 and $2 R_{\odot}$. 


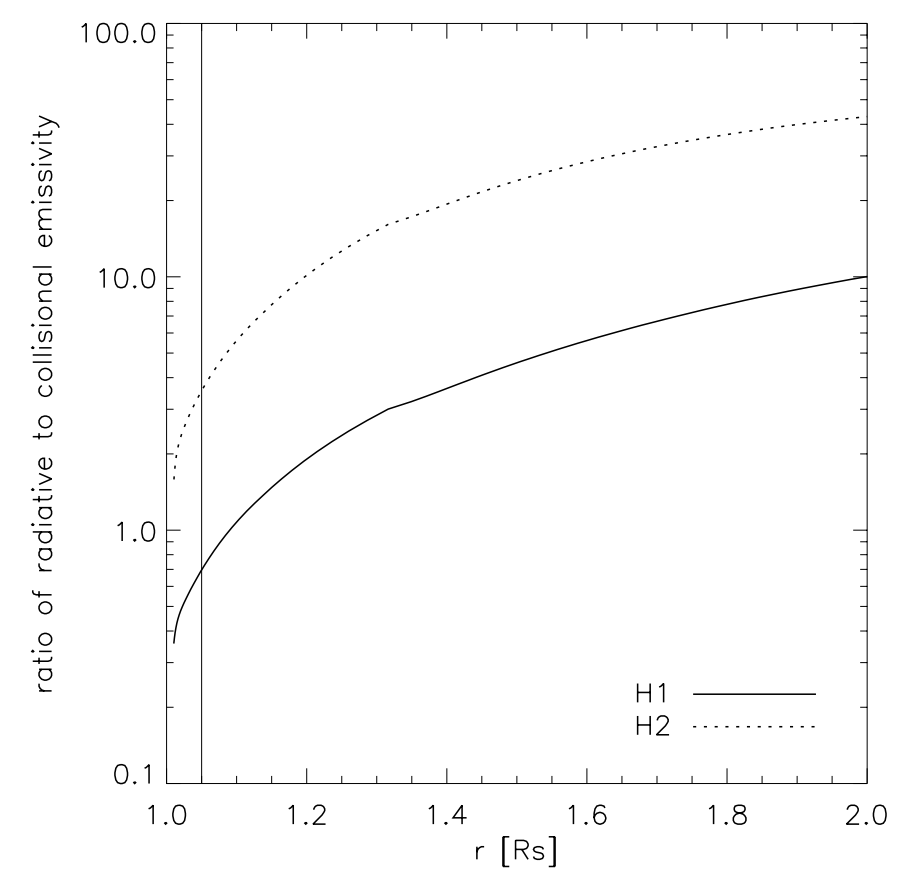

Figure 7. Ratio of radiative to collisional emissivity for $\mathrm{H} 1$ (solid line) and $\mathrm{H} 2$ (dotted line). A vertical black line has been drawn at the height $1.05 R_{\odot}$.

As we can see from Figure 4, the assumption that the falloff of ion and electron densities is the same is not always valid. A Fe buildup in the corona may cause problems when attempting to define $R_{t}$. If the buildup occurs in the region where the $\mathrm{Fe}$ line is dominated by radiative excitation, then the ratio will become constant at some distance, and then begin to increase again, and there is no problem. However, if the buildup occurs in the region where the Fe line is dominated by collisional excitation, or in the transition between the two regions, then there is no way to distinguish between a change in the slope of the line/continuum ratio caused by a transition between the two regions, and a change in the slope caused by a change in the $\mathrm{Fe}$ abundance. Thus, if there is a buildup of $\mathrm{Fe}$ in the corona, the $R_{t}$ as defined by Habbal et al. (2010) may be lower than the "true" $R_{t}$.

In addition, the determination of $R_{t}$ relies on the idea that the line is in the collision-dominated regime at the lowest heights of the observation, because this ensures that we see the very large change in the slope of the line/continuum ratio that occurs below $R_{t}$. If one assumes that the falloff of ion and electron densities is the same, an assumption which ensures that the line/continuum ratio will be constant above $R_{t}$, it would be possible to determine $R_{t}$ even when the line is not in the collision-dominated regime at the lowest heights of the observation. However, the falloff of ion and electron densities is not always the same and we therefore need to see the transition from collisional to radiative excitation in order to get a reasonable estimate for $R_{t}$. In Figure 7 we have plotted the ratio of radiative to collisional emissivity as function of heliocentric distance, $r$, for the Fe XI $789.2 \mathrm{~nm}$ line for both hydrogen backgrounds. Since the ion density, $n_{i}$, is present in both the numerator and denominator, this ratio is independent of the Fe properties and thus Figure 7 applies to all the Fe models described earlier. Note that when an LOS integration is performed, to calculate (or measure) the spectral line intensity, the integrated intensity at height $r$ will be more dominated by radiative excitation than the local emissivity at height $r$, since the integrated intensity includes contributions from higher altitudes, where radiative excitation dominates. The lowest ratio is obtained for the $\mathrm{H} 1$ background, where the ratio is about 0.7 at $1.05 R_{\odot}$ (vertical line in Figure 7 ), which is close to the maximum in the line/continuum ratios in Figure 6 . This means that the line/continuum ratios calculated here are never really in the collision-dominated regime (i.e., a rad/coll ratio below 0.1 ). For the Fe line to be collision-dominated at $1.05 R_{\odot}$, we would have to increase the electron density there by almost an order of magnitude compared to the $\mathrm{H} 1$ background. This would result in densities in accordance with the observations of Uzzo et al. (2007). If such high densities are to be combined with flow velocities large enough that $\mathrm{Fe}$ is driven well out of ionization equilibrium, ensuring that the high charge states survive to $1 \mathrm{AU}$, then the mass flux of the wind would become very large.

If the observed $\mathrm{Fe}$ lines are in the collision-dominated regime below $1.2 R_{\odot}$, as suggested by Habbal et al. (2007), then the electron densities in the structures they have observed should be very high compared to our modeled densities. Although we cannot exclude the possibility that the observed Fe lines really are collision dominated at low heights, it is clear that this cannot be inferred directly from the line/continuum ratio, since the modeled line/continuum ratios shown in Figure 6 all fall off below $1.2 R_{\odot}$, just like the observed ratios. Clearly, this falloff is related to the diminishing collisionally excited component of the line, but the transition occurs over an extended region, enabling us to see a falloff in the line even though it is never dominated by collisional excitation.

Finally, we have seen that the freezing-in height of $\mathrm{Fe}^{+10}$ and $\mathrm{Fe}^{+12}$ is large in all $\mathrm{Fe}$ simulations. The result is that for a lowvelocity background such as $\mathrm{H} 1$, the ion fractions of the high charge states at $1 \mathrm{AU}$ are very small compared to the value of the ion fractions at the height of the electron temperature maximum. In the high-velocity background $\mathrm{H} 2, \mathrm{Fe}$ is pushed further out of ionization equilibrium, and the high charge states are able to survive to larger distances, ensuring a higher ion fraction at $1 \mathrm{AU}$ in these backgrounds. However, if a pileup occurs in the extended corona $\left(4-10 R_{\odot}\right)$, such as in $2 \mathrm{~A}$ and $2 \mathrm{~B}$, the frozen-in ion fractions of the high charge states are significantly reduced.

\section{CONCLUSIONS}

We have found that Fe abundance enhancements, or "pileups," can occur in the slow solar wind for a variety of density and proton temperature profiles. For low proton temperatures in the corona, the existence and location of a pileup depends on the Fe temperature. For high proton temperatures, pileup occurs as a result of low Fe heating rates. In the latter type of hydrogen background, the location and magnitude of the pileup is sensitive to proton temperature and flux density. In both types of background solution, $\mathrm{Fe}$ is able to escape the corona regardless of the amount of Fe heating; hence, high Fe temperatures, of order $100 \mathrm{MK}$, are not required.

We also find that a lack of pileup in $\mathrm{Fe}^{+12}$, where a pileup in $\mathrm{Fe}^{+10}$ is present, can occur either as a result of preferential heating of the higher charge states or because the radial profiles of the electron and proton temperature, density, and flow velocity produce a low $\mathrm{Fe}^{+12}$ density at the height of pileup. The lack of an $\mathrm{Fe}^{+12}$ pileup is not, in general, an indication of the magnitude of the electron temperature maximum.

The synthesized Fe XI $789.2 \mathrm{~nm}$ line/continuum ratios successfully reproduce the observations by Habbal et al. (2007), confirming that the observed "bumps" in the line/continuum 
ratio must be a signature of a local increase in the total density of Fe. By calculating the ratio of the radiative and the collisional emissivity we have determined that for our solar wind solutions, the Fe XI $789.2 \mathrm{~nm}$ line is never fully in the collision-dominated regime. To reach the collision-dominated regime at low heights, the electron density would have to be increased by about an order of magnitude with respect to our highest density hydrogen background solution.

Finally, we find that a high density-low-velocity corona leads to very low ion fractions for $\mathrm{Fe}^{+10}$ and $\mathrm{Fe}^{+12}$ at $1 \mathrm{AU}$, while a low density-high-velocity wind leads to ion fractions in better accordance with observations of Fe charge states in the solar wind (von Steiger et al. 2000). This is mainly a result of the large freezing-in distance of $\mathrm{Fe}$ in the slow solar wind. A Fe pileup in the extended corona $\left(4-10 R_{\odot}\right)$ has a large impact on the frozen-in ion fractions, with the effect of reducing the ion fractions of the high charge states. Our results on the ion fractions of $\mathrm{Fe}^{+10}$ and $\mathrm{Fe}^{+12}$ support the idea that the slow solar wind is rooted in open magnetic field structures with coronalhole-like densities in the low corona, as suggested by Abbo et al. (2010), Antonucci et al. (2005), and Antonucci et al. (2006).

Hanne Sigrun Byhring thanks the Solar, Stellar and Planetary Division at the Harvard-Smithsonain Center for Astrophysics for their hospitality while she was a visitor there. We also thank H. Morgan for providing the data for Figure 5 from Habbal et al. (2007), Figure 9. This work has been supported by a Fulbright Scholarship and by the Norwegian Research Council, grant no. 177570/V30. S.R.C. acknowledges the support of NASA grants NNX09AB27G and NNX10AC11G.

\section{APPENDIX A}

\section{THE MODEL EQUATIONS}

When assuming radial flow, the equations of Janse et al. (2005) reduce to five transport equations for matter, momentum, temperature (parallel and perpendicular to the field), and heat flow, for each particle species, $s$.

The equation of continuity,

$$
\frac{\partial n_{s}}{\partial t}=-\frac{\partial}{\partial r}\left(n_{s} u_{s}\right)-n_{s} u_{s} \frac{1}{A} \frac{\partial A}{\partial r}+\frac{\delta n_{s}}{\delta t},
$$

contains the number density, $n_{s}$, the flow velocity, $u_{s}$, the crosssectional area of the flow tube, $A$, and the production and loss term $\frac{\delta n_{s}}{\delta t}$, which is given by

$$
\frac{\delta n_{s}}{\delta t}=\sum_{t}\left(n_{t} R_{t s}-n_{s} R_{s t}\right)
$$

where $R_{s t}$ is the transition rate from charge state $s$ to charge state $t$ as a result of photoionization, collisional ionization, recombination, and charge exchange.

The equation of motion,

$$
\begin{aligned}
\frac{\partial u_{s}}{\partial t}+u_{s} \frac{\partial u_{s}}{\partial r}= & -\frac{k}{m_{s}} \frac{\partial T_{s \|}}{\partial r}-\frac{k T_{s \|}}{n_{s} m_{s}} \frac{\partial n_{s}}{\partial r} \\
& -\frac{1}{A} \frac{d A}{d r} \frac{k}{m_{s}}\left(T_{s \|}-T_{s \perp}\right)+\frac{e_{s}}{m_{s}} E \\
& -\frac{G M_{\odot}}{r^{2}}-\frac{1}{n_{s} m_{s}} \frac{\partial P_{s w}}{\partial r}+\frac{1}{n_{s} m_{s}} \frac{\delta M_{s}}{\delta t}
\end{aligned}
$$

contains the parallel and perpendicular temperature, $T_{s \|}$ and $T_{s \perp}$, the mass and charge of a particle of species $s, m_{s}$ and $e_{s}$, the Boltzmann constant, $k$, the electric field, $E$, the solar mass, $M_{\odot}$, the gravitational constant, $G$, and the Alfvén wave pressure, $P_{s w}$ (see Lie-Svendsen et al. 2001 for details). The collisional term is given by

$$
\begin{aligned}
\frac{\delta M_{s}}{\delta t} & =-\sum_{t} n_{s} m_{s} v_{s t}\left(u_{s}-u_{t}\right)+\sum_{t} v_{s t} \frac{3}{5} \frac{\mu_{s t}}{k T_{s t}} \\
& \times\left[q_{s}\left(1-\frac{5}{7} \frac{m_{t}}{m_{s}+m_{t}}\right)-q_{t} \frac{m_{s} n_{s}}{m_{t} n_{t}}\left(1-\frac{5}{7} \frac{m_{s}}{m_{s}+m_{t}}\right)\right] \\
& +m_{s} \sum_{t}\left(n_{t} u_{t} R_{t s}-n_{s} u_{s} R_{s t}\right)-\sum_{t} n_{s} m_{s} v_{s t}^{2 s t r}\left(u_{s}-u_{t}\right) .
\end{aligned}
$$

The reduced mass is

$$
\mu_{s t}=\frac{m_{s} m_{t}}{m_{s}+m_{t}}
$$

and the reduced temperature, $T_{s t}$, is defined as

$$
T_{s t}=\frac{T_{s} m_{t}+T_{t} m_{s}}{m_{s}+m_{t}},
$$

where $T_{s}=\left(T_{s \|}+2 T_{s \perp}\right) / 3$ is the average temperature.

The Coulomb collisional frequency is given in Equation (8). The Coulomb logarithm is a factor which accounts for the Debye screening of the particles' electric fields. The expression for the Coulomb logarithm used in the model is

$$
\ln \lambda=23-\ln \left[\left(\frac{n_{e}}{n_{0}}\right)^{1 / 2}\left(\frac{T_{e}}{T_{0}}\right)^{-3 / 2}\right],
$$

where $T_{0}=10^{6} \mathrm{~K}$ and $n_{0}=1 \mathrm{~m}^{-3}$. In the numerical model, ion-neutral and neutral-neutral collisions are also included, with the collision frequency (Banks \& Kockarts 1973)

$$
v_{s t}=\frac{2 \times 10^{-42} \mathrm{~kg} \mathrm{~m}^{3} \mathrm{~s}^{-1}}{m_{s}} n_{t} .
$$

The last term in (Equation (A4)) is introduced to mimic the twostream instability in the outer solar wind. The extra frictional force associated with this term ensures that the drift velocities of protons and iron ions do not deviate by more than the local Alfvén speed, $v_{a}=B / \sqrt{\mu_{0} \rho_{0}}$, as required from in situ measurements (e.g., Marsch 2006, and references therein). The collision frequency $v_{s t}^{2 s t r}$ is given as

$$
\begin{gathered}
v_{s t}^{2 s t r}=\frac{v_{\max }}{2}\left[\tanh \left(\frac{\Delta u_{0}}{\Delta u}\right)+\tanh \left(\frac{\Delta v_{a}-\Delta u_{0}}{\Delta u}\right)\right] \\
v_{\max }=\frac{u_{\text {flow }}}{r_{2 s t r}\left(1+\frac{\rho_{s}}{\rho_{t}}\right)} \\
\Delta v_{a}=\frac{\left|u_{s}-u_{t}\right|}{v_{a}}
\end{gathered}
$$

where $\rho_{0}$ is the total mass density, $\mu_{0}$ is the permeability of vacuum and $u_{\text {flow }}, r_{2 s t r}, \Delta u$ and $\Delta u_{0}$ are constant parameters which have been set to $300 \mathrm{~km} \mathrm{~s}^{-1}, 100 R_{\odot}, 0.2$, and 1 , 
respectively. The dependence on $\tanh \left(\Delta u_{0} / \Delta u\right)$ ensures that the force from this extra friction term is zero when the difference in drift speed is small compared to the Alfvén speed.

The energy conservation equations describe the evolution of the parallel and perpendicular temperature in a frame moving with the fluid:

$$
\begin{gathered}
\frac{\partial T_{s \|}}{\partial t}+u_{s} \frac{\partial T_{s \|}}{\partial r}=-2 T_{s \|} \frac{\partial u_{s}}{\partial r}-\frac{1}{n_{s} k} \frac{\partial q_{s \|}}{\partial r} \\
-\frac{1}{A} \frac{d A}{d r} \frac{q_{s \|}}{n_{s} k}+\frac{2}{A} \frac{d A}{d r} \frac{q_{s \perp}}{n_{s} k}+\frac{1}{n_{s} k} Q_{s m \|}+\frac{1}{n_{s} k} \frac{\delta E_{s \|}}{\delta t}, \\
\frac{\partial T_{s \perp}}{\partial t}+u_{s} \frac{\partial T_{s \perp}}{\partial r}= \\
+\frac{1}{A} \frac{d A}{d r} u_{s} T_{s \perp}-\frac{1}{n_{s} k} \frac{\partial q_{s \perp}}{\partial r}-\frac{2}{A} \frac{d A}{d r} \frac{q_{s \perp}}{n_{s} k} \\
+\frac{1}{n_{s} k} Q_{s m \perp}+\frac{1}{n_{s} k} \frac{\delta E_{s \perp}}{\delta t},
\end{gathered}
$$

where $Q_{s m \|}$ and $Q_{s m \perp}$ describe heating, and the terms $\frac{1}{n_{s} k} \frac{\delta E_{s \perp}}{\delta t}$ and $\frac{1}{n_{s} k} \frac{\delta E_{s \|}}{\delta t}$ are collisional terms given by

$$
\begin{aligned}
\frac{\delta E_{s \|}}{\delta t}= & -\sum_{t} 2 \frac{m_{s}}{m_{s}+m_{t}} n_{s} v_{s t} k\left\{T_{s \|}-T_{t \|}-\frac{2}{5}\left[\frac{m_{t}}{m_{s}}\left(T_{s \perp}-T_{s \|}\right)\right.\right. \\
& \left.\left.+T_{t \perp}-T_{t \|}\right]\right\}+\sum_{t}\left(n_{t} k T_{t \|} R_{t s}-n_{s} k T_{s \|} R_{s t}\right) \\
& +\frac{2}{3} n_{s} \sum_{t} \mu_{s t} v_{s t}\left(u_{s}-u_{t}\right)^{2},
\end{aligned}
$$

$$
\begin{aligned}
\frac{\delta E_{s \perp}}{\delta t}= & -\sum_{t} 2 \frac{m_{s}}{m_{s}+m_{t}} n_{s} v_{s t} k\left\{T_{s \perp}-T_{t \perp}-\frac{1}{5}\left[\frac{m_{t}}{m_{s}}\left(T_{s \perp}-T_{s \|}\right)\right.\right. \\
& \left.\left.+T_{t \perp}-T_{t \|}\right]\right\}+\sum_{t}\left(n_{t} k T_{t \perp} R_{t s}-n_{s} k T_{s \perp} R_{s t}\right) \\
& +\frac{2}{3} n_{s} \sum_{t} \mu_{s t} v_{s t}\left(u_{s}-u_{t}\right)^{2}+n_{s} \sum_{t} \mu_{s t} v_{s t}^{2 s t r}\left(u_{s}-u_{t}\right)^{2} .
\end{aligned}
$$

The radial heat flux density satisfies

$$
\begin{aligned}
\frac{\partial q_{s}}{\partial t}+u_{s} \frac{\partial q_{s}}{\partial r}= & -2 q_{s \|} \frac{\partial u_{s}}{\partial r}-\frac{1}{2} u_{s} q_{s} \| \frac{1}{A} \frac{d A}{d r}-2 q_{s \perp} \frac{\partial u_{s}}{\partial r} \\
& -2 u_{s} q_{s \perp} \frac{1}{A} \frac{d A}{d r}-\frac{k^{2} n_{s} T_{s \|}}{m_{s}} \frac{\partial}{\partial r}\left(\frac{3}{2} T_{s \|}+T_{s \perp}\right) \\
& -\frac{1}{A} \frac{d A}{d r} \frac{k^{2} n_{s} T_{s \perp}}{m_{s}}\left(T_{s \|}-T_{s \perp}\right)+\frac{\delta q_{s}{ }^{\prime}}{\delta t}, \quad \text { (A16) }
\end{aligned}
$$

where

$$
\frac{\delta q_{s}{ }^{\prime}}{\delta t}=\frac{\delta q_{s}}{\delta t}-\frac{k}{m_{s}}\left(\frac{3}{2} T_{s \|}+T_{s \perp}\right) \frac{\delta M_{s}}{\delta t}
$$

and

$$
\begin{aligned}
\frac{\delta q_{s}}{\delta t}= & -\sum_{t \neq s} v_{s t}\left\{E_{s t}{ }^{(1)} q_{s}-E_{s t}{ }^{(4)} \frac{m_{s} n_{s}}{m_{t} n_{t}} q_{t}+\frac{5}{2} p_{s}\left(u_{s}-u_{t}\right)\right. \\
& \left.\times\left[1-\frac{3}{5} \frac{m_{t}}{m_{s}+m_{t}}\right]\right\}-\frac{16}{35} v_{s s} q_{s}+\sum_{t}\left(q_{t} R_{t s}-q_{s} R_{s t}\right),
\end{aligned}
$$

and the mass factors $E_{s t}{ }^{(1)}$ and $E_{s t}{ }^{(4)}$ are defined as

$$
\begin{aligned}
E_{s t}{ }^{(1)} & \equiv \frac{1}{m_{0}{ }^{3}}\left(3 m_{s}{ }^{3}-\frac{1}{2} m_{s}{ }^{2} m_{t}-\frac{2}{5} m_{s} m_{t}{ }^{2}-\frac{4}{35} m_{t}{ }^{3}\right), \\
E_{s t}{ }^{(4)} & \equiv \frac{1}{m_{0}{ }^{3}}\left(\frac{6}{5} m_{t}{ }^{3}-\frac{171}{70} m_{t}{ }^{2} m_{s}-\frac{3}{7} m_{t} m_{s}{ }^{2}\right),
\end{aligned}
$$

where $m_{0}=m_{s}+m_{t}$ and $q_{s}$ is related to the parallel and perpendicular heat fluxes through the equations

$q_{s \|}=30 q_{s} \frac{T_{s \|}{ }^{3}\left(4 T_{s \perp}+3 T_{s \|}\right)}{16 T_{s \perp}{ }^{4}+48 T_{s \perp}{ }^{3} T_{s \|}+6 T_{s \perp}{ }^{2} T_{s \|^{2}}+60 T_{s \perp} T_{s \|^{3}}+45 T_{s \|}{ }^{4}}$

and

$q_{s \perp}=2 q_{s} \frac{T_{s \perp}{ }^{2}\left(8 T_{s \perp}{ }^{2}+24 T_{s \perp} T_{s \|}+3 T_{s \|}{ }^{2}\right)}{16 T_{s \perp}{ }^{4}+48 T_{s \perp}{ }^{3} T_{s \|}+6 T_{s \perp}{ }^{2} T_{s \|^{2}}+60 T_{s \perp} T_{s \|}{ }^{3}+45 T_{s \|}{ }^{4}}$.

The transport of energy occurs strictly parallel to the magnetic field. The perpendicular heat flow is a flow of perpendicular thermal energy, in the direction parallel to the magnetic field.

The cross-sectional area of the flow tube is given by (Kopp \& Holzer 1976)

$$
A(r)=A_{0}\left(\frac{r}{R_{S}}\right)^{2} f_{1}(r) f_{2}(r)
$$

where $A_{0}=1 \mathrm{~m}^{2}$ is the area of the flow tube at the solar surface and

$$
f_{i}(r)=\frac{f_{\max _{i}} \exp \left[\frac{r-R_{1 i}}{\sigma_{i}}\right]+f_{1 i}}{\exp \left[\frac{r-R_{1 i}}{\sigma_{i}}\right]+1}
$$

where

$$
f_{1_{i}}=1-\left(f_{\max _{i}}-1\right) \exp \left[\frac{R_{\odot}-R_{1 i}}{\sigma_{i}}\right] .
$$

$f_{i}(r)$ is the expansion function and describes the expansion of the flow tube as a function of radial distance. The expression for the cross-sectional area of the flow tube allows for nonradial expansion in two different regions along the flow tube, around $R_{11}$ and $R_{12}$. At $R_{11}\left(R_{12}\right)$, the cross-sectional area of the flow tube increases to $f_{\max 1}\left(f_{\max 1} f_{\max 2}\right)$ times the area of a radially expanding flow tube, and most of the increase occurs in the region $R_{11}-\sigma_{1}$ to $R_{11}+\sigma_{1}\left(R_{12}-\sigma_{2}\right.$ to $\left.R_{12}+\sigma_{2}\right)$. The maximum value of $\frac{\partial f_{i}}{\partial r}$ occurs at $R_{1 i}$. In our hydrogen background models, $f_{\max 1}=14, f_{\max 2}=7, R_{11}=1.017 R_{S}$, $R_{12}=1.3 R_{S}, \sigma_{1}=0.004 R_{S}$, and $\sigma_{2}=0.5 R_{S}$.

\section{APPENDIX B}

\section{Fe XI LINE SYNTHESIS}

The spectral line intensity at a radial distance $r=r^{\prime}$ was calculated by integrating the sum of the collisional and radiative emissivity along the LOS, which is assumed to be perpendicular to the radial direction. The integration was done over a region of $60 R_{\odot}$, centered on the point $r^{\prime}$. We assume a spherically symmetric atmosphere so that the parameters at a distance $L$ 
(where $L$ varies between 0 and $30 R_{\odot}$ ) from the point $r=r^{\prime}$ can be approximated by the plasma parameters at $r=\sqrt{r^{\prime 2}+L^{2}}$.

The frequency-dependent collisional emissivity can be expressed in terms of the ion and electron densities, $n_{i}$ and $n_{e}$, as

$$
j_{\mathrm{coll}, v}=\frac{h v_{0}}{4 \pi} q_{i j}\left(T_{e}\right) n_{e} n_{i} \phi(v),
$$

where $v_{0}$ is the line-center frequency in vacuum and $h$ is the Planck constant. Assuming a Maxwellian velocity distribution, the line profile function, $\phi(v)$, is

$$
\phi(v)=\frac{1}{\Delta v_{D} \sqrt{\pi}} \exp \left[-\left(\frac{v-v_{0}}{\Delta v_{D}}\right)^{2}\right]
$$

with $\Delta v_{D}=v_{0} v_{\text {th }} / c$, where $v_{\text {th }}$ is the ion perpendicular thermal speed and $c$ is the speed of light. By integrating over wavelength we obtain the frequency-independent emissivity

$$
j_{\mathrm{coll}}=\frac{h v_{0}}{4 \pi} q_{i j}\left(T_{e}\right) n_{e} n_{i}
$$

The temperature-dependent excitation rate between levels $i$ and $j, q_{i j}\left(T_{e}\right)$, can be written as

$$
q_{i j}=C_{i j} \exp \left[\frac{-h v_{0}}{k T_{e}}\right],
$$

where $k$ is the Boltzmann constant, $T_{e}$ the electron temperature, and $C_{i j}$ is defined as (Bhatia \& Doschek 1996)

$$
C_{i j}=\frac{8.63 \times 10^{-6}}{g_{i} \sqrt{T_{e}}} \cdot \Upsilon_{i j}
$$

The factor $g_{i}$ is the statistical weight assigned to level $i$ and $\Upsilon_{i j}$, the effective collision strength, is a function of electron temperature and is tabulated in Aggarwal \& Keenan (2003). For Fe XI $\lambda 7892, \Upsilon_{i j}$ ranges from 2.26 at $T_{e}=10^{4} \mathrm{~K}$ to 0.56 at $T_{e}=5 \times 10^{6} \mathrm{~K}$.

The frequency-dependent radiative emissivity is

$$
j_{\mathrm{rad}, v}=\frac{h \nu_{0}}{4 \pi} n_{i} B_{i j} \iint I_{\nu^{\prime}} R_{I} \frac{d \Omega^{\prime}}{4 \pi} d \nu^{\prime},
$$

where $B_{i j}$ is the rate of direct absorption of photons, $I_{v^{\prime}}$ is the intensity profile from the solar disk, and $R_{I}$ is the redistribution function. The integral over $d \Omega^{\prime}$ can be approximated by the "dilution factor," $W=0.5\left(1-\sqrt{1-\left(R_{S}^{2} / r^{2}\right)}\right)$. The intensity profile can be approximated by

$$
I_{v^{\prime}}=I_{\text {cont }}-I_{\text {abs }} \exp \left[-\left(\frac{v-v_{0}}{\Delta v_{I}}\right)^{2}\right] \text {. }
$$

The parameters $I_{\text {cont }}, I_{\text {abs }}$, and $\Delta v_{I}$ can be obtained from the solar flux atlas of Kurucz et al. (1984). For Fe XI $\lambda 7892$, $I_{\text {cont }} \approx 4.4 \times 10^{-8} \mathrm{~W}\left(\mathrm{~m}^{2} \mathrm{sr} \mathrm{Hz}\right)^{-1}, I_{\mathrm{abs}} \approx 1.1 \times 10^{-8} \mathrm{~W}$ $\left(\mathrm{m}^{2} \mathrm{sr} \mathrm{Hz}\right)^{-1}$, and $\Delta v_{I} \approx 0.18 \mathrm{~nm}$.

The redistribution function, assuming a Maxwellian velocity distribution function and isotropic, coherent, pure right angle scattering from idealized two-level atoms, can be written as (e.g., Cranmer 1998)

$$
R_{I}=\frac{1}{\Delta v_{D}^{2} \pi} \exp \left[-\left(\frac{v^{\prime}-v_{0}}{\Delta v_{D}}\right)^{2}\right] \exp \left[-\left(\frac{v-v_{0}}{\Delta v_{D}}\right)^{2}\right]
$$

This expression assumes that "Doppler dimming" due to the solar wind flow is negligible. Integrating the frequency-dependent emissivity over wavelength we obtain

$$
j_{\mathrm{rad}}=\frac{h v_{0}}{4 \pi} B_{i j} n_{i} W\left(I_{\mathrm{cont}}-I_{\mathrm{abs}} \frac{\Delta v_{I}}{\sqrt{\Delta v_{I}^{2}+\Delta v_{D}^{2}}}\right) .
$$

An accurate calculation of the continuum emissivity would involve an integral over the photospheric absorption spectrum and over the velocity distribution function of the coronal electrons (Cram 1976). Since we are primarily interested in the radial evolution of the line/continuum ratio, rather than its absolute value, we have chosen a simpler approach by assuming that the continuum emissivity is proportional to $n_{e}$.

We wish to compare the calculated line/continuum ratios with those shown in Habbal et al. (2007). In that paper, the line/ continuum ratios were normalized by dividing with the mean ratio along the radial path. In principle, it would be possible to normalize the calculated line/continuum ratio in the same way, so as to be able to compare the calculated and observed ratios directly. However, the length of each radial path, along which the line/continuum ratio was observed, varies, which means that the choice of normalization constant employed by Habbal et al. (2007) is actually somewhat arbitrary. To compare the magnitude of the calculated line/continuum ratios with the observed ones is, therefore, not straightforward. For this reason, we have chosen to normalize the calculated line/continuum ratios to 1 .

\section{REFERENCES}

Abbo, L., Antonucci, E., Mikić, Z., Linker, J. A., Riley, P., \& Lionello, R. 2010, Adv. Space Res., 46, 1400

Aggarwal, K. M., \& Keenan, F. P. 2003, A\&A, 399, 799

Antonucci, E., Abbo, L., \& Dodero, M. A. 2005, A\&A, 435, 699

Antonucci, E., Abbo, L., \& Telloni, D. 2006, ApJ, 643, 1239

Banaszkiewicz, M., Axford, W. I., \& McKenzie, J. F. 1998, A\&A, 337, 940

Banks, P. M., \& Kockarts, G. (ed.) 1973, Aeronomy (New York: Academic)

Bhatia, A. K., \& Doschek, G. A. 1996, At. Data Nucl. Data Tables, 64, 183

Chen, Y., Esser, R., \& Hu, Y. 2003, ApJ, 582, 467

Cram, L. E. 1976, Sol. Phys., 48, 3

Cranmer, S. R. 1998, ApJ, 508, 925

Cranmer, S. R. 2009, Living Rev. Sol. Phys., 6, 3

Cranmer, S. R., van Ballegooijen, A. A., \& Edgar, R. J. 2007, ApJS, 171, 520

Cranmer, S. R., et al. 1999, ApJ, 511, 481

Doschek, G. A. 1997, ApJ, 476, 903

Dowdy, J. F., Jr., Rabin, D., \& Moore, R. L. 1986, Sol. Phys., 105, 35

Esser, R., Edgar, R. J., \& Brickhouse, N. S. 1998, ApJ, 498, 448

Esser, R., Leer, E., Habbal, S. R., \& Withbroe, G. L. 1986, J. Geophys. Res., 91,2950

Guhathakurta, M., Fludra, A., Gibson, S. E., Biesecker, D., \& Fisher, R. 1999, J. Geophys. Res., 104, 9801

Habbal, S. R., Morgan, H., Johnson, J., Arndt, M. B., Daw, A., Jaeggli, S., Kuhn, J., \& Mickey, D. 2007, ApJ, 663, 598

Habbal, S. R., et al. 2010, ApJ, 708, 1650

Hollweg, J. V., \& Isenberg, P. A. 2002, J. Geophys. Res.: Space Phys., 107, 1147

Hundhausen, A. J., Gilbert, H. E., \& Bame, S. J. 1968, J. Geophys. Res., 73, 5485

Janse, Å. M., Lie-Svendsen, Ø., \& Leer, E. 2005, J. Plasma Phys., 71, 611

Judge, P. G., \& Meisner, R. W. 1994, in Solar Dynamic Phenomena and Solar Wind Consequences, The Third SOHO Workshop, ed. J. J. Hunt (ESA SP-373; Noordwijk: ESA), 67

Ko, Y.-K., Fisk, L., Geiss, J., Gloeckler, G., \& Guhathakurta, M. 1997, Sol. Phys., 171, 345

Kopp, R., \& Holzer, T. 1976, Sol. Phys., 49, 43

Kurucz, R. L., Furenlid, I., Brault, J., \& Testerman, L. 1984, Solar Flux Atlas from 296 to $1300 \mathrm{~nm}$ (Sunspot, NM: National Solar Observatory)

Lie-Svendsen, Ø., \& Esser, R. 2005, ApJ, 618, 1057

Lie-Svendsen, Ø., Hansteen, V. H., Leer, E., \& Holzer, T. E. 2002, ApJ, 566, 562 
Lie-Svendsen, Ø., Leer, E., \& Hansteen, V. H. 2001, J. Geophys. Res., 106, 8217

Marsch, E. 2006, Living Rev. Sol. Phys., 3, 1

Raymond, J. C., Ciaravella, A., Dobrzycka, D., Strachan, L., Ko, Y., Uzzo, M., \& Raouafi, N. 2003, ApJ, 597, 1106

Seely, J. F., Feldman, U., Schuehle, U., Wilhelm, K., Curdt, W., \& Lemaire, P. 1997, ApJ, 484, L87
Uzzo, M., Ko, Y., \& Raymond, J. C. 2004, ApJ, 603, 760

Uzzo, M., Strachan, L., \& Vourlidas, A. 2007, ApJ, 671, 912

Uzzo, M., Strachan, L., Vourlidas, A., Ko, Y., \& Raymond, J. C. 2006, ApJ, 645,720

von Steiger, R., et al. 2000, J. Geophys. Res., 105, 27217

Wilhelm, K., Marsch, E., Dwivedi, B. N., Hassler, D. M., Lemaire, P., Gabriel, A. H., \& Huber, M. C. E. 1998, ApJ, 500, 1023 